\title{
Circunstâncias e consequências do cuidar: estudo prospetivo em cuidados paliativos
}

\author{
Circumstances and consequences of care: \\ a prospective study in palliative care
}

Mayra Delalibera ${ }^{1}$ Alexandra Coelho ${ }^{2}$ Joana Presa ${ }^{2}$ António Barbosa ${ }^{3}$ Isabel Leal ${ }^{1}$
${ }^{1}$ Instituto Superior de Psicologia Aplicada. R. Jardim do Tabaco 34. 1149041 Lisboa Portugal. mayrarmani@yahoo.com.br ${ }^{2}$ Unidade de Medicina Paliativa, Centro Hospitalar Lisboa Norte, Hospital de Santa Maria. Lisboa Portugal.

${ }^{3}$ Faculdade de Medicina, Universidade de Lisboa. Lisboa Portugal.

\begin{abstract}
The sundry circumstances that affect end-of-life care can have a determining role in the result of this experience, particularly in psychological morbidity levels before and after bereavement. The aim of this study is to describe the prevalence of psychopathological symptoms and overburden among family caregivers in palliative care and to identify the circumstances associated with care provision. An attempt was made to evaluate the progress of psychopathological symptoms, identifying circumstantial factors that can interfere in acute grief. The sample consisted of 75 Portuguese caregivers, mostly women and patients' daughters. The results show that caregivers more involved with patient care bear a greater burden and are more likely to manifest psychopathological symptoms, which persist in acute grief. Moreover, the existence of peritraumatic symptoms seem to contribute significantly to the overall state of distress in acute grief. Social support has a protective effect in the different symptomatologic situations and are closely related to family dynamics. These results suggest the possibility of early identification of the most vulnerable caregivers and the adverse circumstances that affect them.
\end{abstract}

Key words Family caregivers, Palliative care, Overburden, Psychological morbidity, Grief
Resumo As várias circunstâncias que afetam a prestação de cuidados em fim-de-vida podem ter um papel determinante no resultado desta experiência, nomeadamente nos níveis de morbilidade psicológica antes e depois da perda. Este estudo tem por objetivos descrever a prevalência da sobrecarga e sintomatologia psicopatológica dos cuidadores familiares em cuidados paliativos e identificar as circunstâncias que the estão associadas. Pretendemos ainda avaliar a evolução da sintomatologia, identificando os fatores circunstanciais que interferem na fase aguda do luto. A amostra foi constituída por 75 cuidadores portugueses, a maioria do sexo feminino e filhas do doente. Os resultados revelam que as pessoas mais envolvidas nos cuidados ao doente apresentam mais sobrecarga e estão mais propensas a sintomatologia psicopatológica, a qual tem tendência a manter-se no luto. Ficou também demonstrada a existência de sintomas peritraumáticos que parecem contribuir significativamente para o estado geral de distress no luto agudo. O suporte social tem efeito protetor nos diferentes quadros sintomatológicos e está relacionado com o funcionamento familiar. Estes resultados sugerem a possibilidade de identificar precocemente os cuidadores mais vulneráveis e as circunstâncias adversas que os afetam.

Palavras-chave Cuidadores familiares, Cuidados paliativos, Sobrecarga, Morbilidade psicológica, Luto 


\section{Introdução}

Cuidar de um familiar com uma doença terminal representa uma considerável sobrecarga, tendo em conta a exigência física e emocional inerente a este papel ${ }^{1}$, muitas vezes desempenhado sem preparação prévia e em sobreposição com outras tarefas ${ }^{2}$. Acarreta, por isso, diversas consequências ao nível da morbilidade física e psicológica ${ }^{3,4}$, incluindo sintomas de depressão e ansiedade ${ }^{5,6}$.

Sabe-se, no entanto, que a experiência do cuidar é mediada por diversas circunstâncias, entre as quais, o tempo de prestação de cuidados ${ }^{7}$ e a intensidade do cuidar ${ }^{8}$. A estas dimensões objetivas da sobrecarga, acresce o distress inerente à antecipação da perda, que remete para aspectos como a percepção da evolução doença ${ }^{5}$ e a falta de preparação para a morte 9 .

$\mathrm{O}$ efeito negativo da experiência do cuidar pode, no entanto, ser compensado pelo suporte de outras pessoas ${ }^{10}$, bem como o acesso a recursos sociais e profissionais ${ }^{11}$. Todavia, há evidência de que o suporte informal e profissional não é considerado suficiente na fase de prestação de cuidados ${ }^{12}$.

Sendo a família o principal provedor de cuidados ao doente em fim de vida, a sua dinâmica constitui um elemento determinante no processo de adaptação à doença e perda ${ }^{13,14}$. Kissane et al. ${ }^{13}$ classificam as famílias como funcionais, intermédias e disfuncionais, distinguindo-as em cinco tipologias, consoante os seus níveis de coesão, expressividade e conflito. Dentro das famílias funcionais estão as famílias apoiadoras e as solucionadoras de conflito e as famílias disfuncionais são compostas pelas famílias mal-humoradas e hostis. As famílias disfuncionais têm maiores níveis de morbilidade psicossocial, incluindo depressão. Das famílias dos doentes em cuidados paliativos, 15 a $20 \%$ são consideradas disfuncionais ${ }^{3,15}$.

Os dados da literatura sugerem a continuidade dos níveis de sintomatologia dos cuidadores no período subsequente à morte ${ }^{16}$, ou seja, aqueles sujeitos que mais sintomatologias apresentam na fase de prestação de cuidados são também os que mais dificuldades de adaptação apresentam numa fase inicial do luto. Este facto suporta a hipótese de que o efeito cumulativo da sobrecarga do cuidador e do distress relacionado com a antecipação da morte se reflete na fase posterior à perda. Há mesmo evidência de sintomatologia de stress pós-traumático que se manifesta na linguagem do impacto, incerteza e impotência do cuidar, bem como nas memórias intrusivas da degradação do doente ${ }^{17}$.
Dada a inexistência de resultados relativos à população portuguesa de cuidadores familiares propomo-nos, neste estudo, descrever a prevalência da sobrecarga do cuidador e sintomatologia psicopatológica dos cuidadores familiares em cuidados paliativos e identificar as circunstâncias que lhe estão associadas. Além disso, tratando-se de um estudo prospectivo temos, como segundo objetivo, avaliar a evolução dos sintomas psicopatológicos, identificando os fatores circunstanciais que interferem na fase aguda do luto.

\section{Metodologia}

\section{Participantes}

A amostra, selecionada por conveniência, foi constituída por cuidadores familiares de doentes oncológicos acompanhados na Unidade de Medicina Paliativa do Hospital de Santa Maria - Centro Hospital Lisboa Norte. Foram considerados os seguintes critérios de exclusão: indivíduos com idade inferior a 18 anos, com comprometimento cognitivo ou perturbação física e/ou mental que dificulte a capacidade de responder aos instrumentos, e não domínio da língua portuguesa. Os participantes foram informados sobre o objetivo do estudo e foi solicitado o consentimento informado.

\section{Instrumentos}

Foi utilizado um breve questionário desenvolvido pelos autores para a caracterização sociodemográfica do cuidador familiar e do doente, incluindo também algumas questões estruturadas relativas à percepção da evolução da doença, ao envolvimento no cuidar e preparação subjetiva para a morte.

A sobrecarga foi avaliada através do questionário de sobrecarga do cuidador (Zarit Burden Interview) validado para a população portuguesa por Ferreira et al. ${ }^{18}$, a escala contém 22 itens com pontuações que variam de 0 (nunca) até 4 (sempre).

Para avaliar a sintomatologia psicopatológica foram utilizadas as subescalas de ansiedade, depressão e somatização do Inventário de Sintomas Psicopatológicos (BSI) de Derogatis e Melisaratos $^{19}$, validado para a população portuguesa por Canavarro $^{20}$. O instrumento é composto por 19 afirmações e as respostas variam entre 0 (nunca) e 4 (sempre).

O funcionamento familiar foi avaliado através da versão reduzida do FRI (Family Relationships 
Index) da autoria de Kissane et al. ${ }^{21}$, traduzido e validado para a população portuguesa por Delalibera et al. ${ }^{22}$. O FRI reduzido é composto por 12 itens, e as respostas são dicotómicas ( A escala classifica as famílias em cinco categorias: apoiantes, solucionadoras de conflito, intermédias, mal-humoradas e hostis. Para avaliar esta variável acrescentámos quatro questões estruturadas que incidem sobre as alterações na dinâmica familiar, existência de conflitos e alterações na relação com o doente na fase atual de doença.

O suporte social foi avaliado pela Escala de Provisões Sociais (EPS), versão portuguesa de Moreira e Canaipa ${ }^{23}$. A escala é constituída por 24 itens, pontuados numa escala Likert, com valores que variam de 1 (discordo fortemente) a 4 (concordo fortemente) e os resultados podem ser apresentados em dois grandes fatores: apoio íntimo e apoio casual. Foram também acrescentadas duas questões estruturadas que avaliam o apoio prático e emocional percepcionado pelos cuidadores na fase de doença.

No segundo momento de avaliação foi utilizado o Questionário de Experiências Dissociativas Peritraumáticas (QEDP - Peritraumatic Dissociative Experiences Questionnaire) da autoria de Marmar et al. ${ }^{24}$ para avaliar a experiência de dissociação peritraumática. O questionário foi validado para a população portuguesa por Maia et $\mathrm{al}^{25}$, é composto por 10 itens que avaliam respostas dissociativas durante a exposição a um acontecimento potencialmente traumático. As respostas são fornecidas numa escala tipo Likert de 5 pontos $(1=$ nada verdade a $5=$ extremamente verdade).

\section{Procedimentos}

A presente investigação contou com dois momentos de avaliação. O primeiro momento (T0) decorreu durante a fase de prestação de cuidados, enquanto o doente estava a ser acompanhado na Unidade de Cuidados Paliativos. Neste momento foram avaliadas as variáveis sociodemográficas, o processo de doença e prestação de cuidados, a sintomatologia psicopatológica, a sobrecarga do cuidador, a dinâmica e o funcionamento familiar e o suporte social. Algumas informações referentes ao doente - tempo de doença, data do diagnóstico e admissão na equipa de cuidados paliativos - foram recolhidas do processo clínico do doente.

O segundo momento de avaliação (T1) decorreu entre o primeiro e o segundo mês após o óbito do doente. No T1 foram avaliadas as alte- rações na dinâmica familiar, no suporte social, a sintomatologia psicopatológica, as circunstâncias de morte, a preparação subjetiva para a morte e as experiências dissociativas peritraumáticas referentes à perda de um familiar. Solicitámos também a avaliação retrospectiva da sobrecarga do cuidador, replicando a última questão da escala de Zarit "De um modo geral, até que ponto se sentiu sobrecarregado/a por tomar conta do seu familiar?".

Os cuidadores que concordaram em participar no estudo puderam optar por preencher os questionários em papel (presencialmente, levar para casa e entregar num outro dia ou devolver por correio), ou eletronicamente (através de um questionário online). No T1 primeiramente procedeu-se um contato telefónico no qual eram manifestadas as condolências e solicitada a participação na continuidade do estudo. Mais uma vez os participantes puderam optar pelo preenchimento online ou em papel.

A recolha de dados ocorreu de março de 2014 a dezembro de 2015. Durante esse período 130 cuidadores familiares aceitaram participar do estudo, mas apenas 75 responderam integralmente ao questionário, oito foram excluídos por não responderem ao questionário completo e 47 nunca chegaram a entregar os questionários devido ao falecimento imediato do doente, alta da equipa ou falta de disponibilidade.

Dos 75 participantes que responderam ao T0, apenas 29 responderam ao T1. Até ao final de dezembro, 17 doentes ainda estavam a ser acompanhados pela equipa de cuidados paliativos, 17 tiveram alta (foram transferidos para outros serviços de cuidados paliativos) e dos 41 que faleceram, não conseguimos entrar em contato com nove familiares na fase do luto. Dos 32 familiares que conseguimos contatar e concordaram em responder à segunda fase da investigação, 29 devolveram o questionário preenchido e três não responderam.

O presente estudo foi aprovado pela Comissão de Ética do Centro de Investigação do Instituto Superior de Psicologia Aplicada e também pela Comissão de Ética do Hospital de Santa Maria em 24 de outubro de 2014.

\section{Análise dos dados}

Para a análise dos dados recolhidos foi utilizado o programa estatístico SPSS versão 22.0. Realizaram-se análises descritivas e de frequências para estabelecer a prevalência das variáveis em estudo (sociodemográficas, sobrecarga, dinâmi- 
ca familiar, suporte social, ansiedade, depressão e somatização), utilizou-se as correlações de Spearman para avaliar as correlações entre as variáveis ordinais e entre ordinais e quantitativas, e as correlações de Pearson para examinar a relação entre variáveis quantitativas. Para analisar se uma variável quantitativa apresentava diferenças entre grupos foram utilizados os testes t-student e o não paramétrico Wilcoxon-Mann-Witney (quando as variáveis não cumpriam os pressupostos de normalidade e homogeneidade). Para a comparação entre as variáveis avaliadas no T0 e T1 (sobrecarga, ansiedade, depressão e somatização) utilizou-se o teste t-student para amostras emparelhadas. Verificou-se a normalidade das variáveis através dos coeficientes de assimetria e curtose. Os valores de assimetria foram considerados adequados quando inferiores a três e os de curtose quando inferiores a sete. Os resultados foram considerados estatisticamente significativos para $\mathrm{p} \leq 0,05$.

\section{Resultados}

\section{Características sociodemográficas}

A amostra do estudo foi constituída por 75 cuidadores familiares de doentes oncológicos acompanhados em cuidados paliativos. Os cuidadores eram, na maioria, do sexo feminino $(81,3 \%)$, casados $(77,3 \%)$, filhos $(60 \%)$ e com uma média de idades de 51,95 anos ( $\mathrm{DP}=13,41)$ (Tabela 1). Os doentes eram maioritariamente do sexo masculino $(53,3 \%)$, com uma média de idades de 69,81 anos ( $\mathrm{DP}=13,18)$ (Tabela 1). A maioria (48\%) dos doentes foi diagnosticado entre um e cinco anos e $29,3 \%$ foi acompanhado

Tabela 1. Caracterização sociodemográfica da amostra.

\begin{tabular}{|c|c|c|}
\hline & Participantes $(\mathrm{N}=75)$ & Doentes acompanhados em $\mathrm{CP}(\mathrm{N}=75)$ \\
\hline Idade Média (Desvio Padrão) & $51,94(\mathrm{DP}=13,41)$ & $69,81(\mathrm{DP}=13,18)$ \\
\hline Amplitude & $18-79$ & $24-97$ \\
\hline \multicolumn{3}{|l|}{ Sexo, n (\%) } \\
\hline Masculino & $14(18,7)$ & $40(53,3)$ \\
\hline Feminino & $61(81,3)$ & $35(46,7)$ \\
\hline \multicolumn{3}{|l|}{ Raça/ etnia, n (\%) } \\
\hline Caucasiana & $73(93,4)$ & \\
\hline Negra & $1(1,3)$ & \\
\hline Asiática & $1(1,3)$ & \\
\hline \multicolumn{3}{|l|}{ Estado Civil, n (\%) } \\
\hline Solteiro & $12(16)$ & \\
\hline Casado/ União estável & $58(77,3)$ & \\
\hline Separado/ Divorciado & $5(6,7)$ & \\
\hline \multicolumn{3}{|l|}{ Escolaridade, $\mathrm{n}(\%)$} \\
\hline $1^{\circ}$ ciclo & $\begin{array}{r}0(10,0) \\
3(4)\end{array}$ & \\
\hline $2^{\circ}$ ciclo & $15(20)$ & \\
\hline $3^{\circ}$ ciclo & $18(24)$ & \\
\hline Secundário & $\begin{array}{r}18(24) \\
5(6,7)\end{array}$ & \\
\hline Curso tecnológico & $22(29,3)$ & \\
\hline $\begin{array}{l}\text { Licenciatura } \\
\text { Mestrado }\end{array}$ & $4(5,3)$ & \\
\hline \multicolumn{3}{|l|}{ Situação Profissional, n (\%) } \\
\hline Ativo & $38(50,7)$ & \\
\hline Baixa médica & $4(5,3)$ & \\
\hline Desempregado & $9(12)$ & \\
\hline Reformado & $18(24)$ & \\
\hline Não trabalha / doméstica & $6(8)$ & \\
\hline \multicolumn{3}{|l|}{ Parentesco, n (\%) } \\
\hline Cônjuge & $25(33,3)$ & \\
\hline Filho (a) & $45(60)$ & \\
\hline Pai/ Mãe & $2(2,7)$ & \\
\hline Outros familiares & $3(4)$ & \\
\hline
\end{tabular}


pelo serviço de cuidados paliativos entre três e seis meses (Tabela 2).

\section{Dados obtidos na fase de prestação de cuidados (T0)}

Prevalência da sobrecarga do cuidador e sintomatologia psicopatológica - O nível de sobrecarga do cuidador avaliado através da Zarit identificou que $52 \%$ dos cuidadores apresentavam uma sobrecarga moderada, $26,7 \%$ sobrecarga moderada a severa e $20 \%$ dos cuidadores não apresentavam sinais de sobrecarga. A maioria dos cuidadores apresentou níveis de ansiedade (61,3\%), depressão $(69,3 \%)$ e somatização $(54,7 \%)$ acima da média estabelecida por Canavarro ${ }^{20}$ para a população portuguesa.

Descrição dos fatores situacionais da fase do cuidar - No que diz respeito ao processo de diagnóstico do doente, $38,7 \%$ dos cuidadores referiram que o diagnóstico foi rápido e $65,3 \%$ não estavam nada à espera desse diagnóstico. Em 56,8\% dos casos a evolução da doença foi rápida ou muito rápida e a maioria $(42,5 \%)$ dos cuidadores não estava nada à espera que a doença avançasse tão rapidamente. No momento em que responderam ao estudo $79,4 \%$ dos cuidadores referiram que o estado de saúde do seu familiar era mau ou muito mau (Tabela 3 ).

A maioria $(68 \%)$ dos cuidadores coabitava com o doente, $36 \%$ estavam envolvidos nos cuidados ao doente há mais de dois anos, e 41,3\% dedicavam mais de 16 horas do seu dia a cuidar do seu familiar. Além disso, 37,3\% dos cuidadores já tinham cuidado de um outro familiar doente antes.

A maioria $(74,3 \%)$ dos cuidadores familiares referiu ter apoio de outras pessoas, principalmente de outros familiares $(53,3 \%)$. No entanto, 40,5\% não tinham todo o apoio prático de que necessitavam para poder cuidar adequadamente do doente e quando questionados relativamente ao apoio emocional, 51,4\% relataram ter com quem conversar, mas preferiam não o fazer para não preocupar as outras pessoas com os seus problemas. Os dados descritivos que apresentamos referem-se aos resultados das questões estruturadas sobre o apoio prático e emocional (Tabela 3).

$\mathrm{Na}$ resposta às questões estruturadas sobre as alterações na dinâmica familiar, a maioria dos participantes relatou que, desde que o familiar adoeceu, os demais familiares estavam mais próximos, tanto na família nuclear $(40,5 \%)$ quanto na família alargada $(37,8 \%)$. De acordo com a

Tabela 2. Diagnóstico e tempo de acompanhamento em Cuidados Paliativos.

\begin{tabular}{lc}
\hline & Doentes acompanhados em $\mathbf{C P}(\mathbf{N}=\mathbf{7 5})$ \\
\hline Diagnóstico & $\mathbf{n}(\%)$ \\
Neoplasia das vias biliares, fígado e pâncreas & $13(17,3)$ \\
Neoplasia do trato intestinal & $10(13,3)$ \\
Neoplasia do rim e estruturas anexas & $10(13,3)$ \\
Neoplasia do sistema gastresofágico & $7(9,4)$ \\
Neoplasia da mama & $7(9,4)$ \\
Neoplasia de cabeça e pescoço & $6(8)$ \\
Neoplasia da próstata & $6(8)$ \\
Neoplasia de Pulmão & $4(5,3)$ \\
Outras neoplasias & $12(16)$ \\
Tempo de diagnóstico & \\
Menos de 6 meses & $14(18,6)$ \\
Mais de 6 meses a 1 ano & $12(16)$ \\
Mais de 1 a 3 anos & $18(24)$ \\
Mais de 3 a 5 anos & $18(24)$ \\
Mais de 5 a 7 anos & $8(10,7)$ \\
Mais de 7 anos & $5(6,7)$ \\
Tempo de acompanhamento em CP & \\
Até 1 mês & $11(14,7)$ \\
De 1 a 3 meses & $19(25,3)$ \\
Mais de 3 a 6 meses & $22(29,3)$ \\
Mais de 6 meses a 1 ano & $17(22,7)$ \\
Mais de 1 ano & $6(8)$ \\
\hline
\end{tabular}


classificação das tipologias familiares do FRI a maioria dos participantes $(29,3 \%)$ foram classificados como famílias intermédias, seguido da tipologia familiar mal-humorada (24\%) e das solucionadoras de conflito $(22,7 \%)$ (Tabela 3$)$.
Fatores relacionados com a sobrecarga e sintomatologia psicopatológica - Os cuidadores que referiram não ter apoio de outras pessoas apresentaram valores mais elevados de sobrecarga do que os cuidadores que tinham apoio, e as dife-

Tabela 3. Variáveis dependentes e independentes avaliadas no T0.

\begin{tabular}{|c|c|c|c|}
\hline Variável & $\mathrm{N}=75$ & Variável & $\mathrm{N}=75$ \\
\hline Estado de saúde atual do doente & n (\%) & Apoio prático & n (\%) \\
\hline Muito mal & $23(31,5)$ & Não tenho apoio & $19(25,7)$ \\
\hline Mau & $35(47,9)$ & Tenho algum apoio (insuficiente) & $30(40,5)$ \\
\hline Razoável & $15(20,6)$ & Não necessito de mais apoio & $25(33,8)$ \\
\hline Processo de diagnóstico & & Apoio emocional & \\
\hline Muito lento & $8(11,1)$ & Não tenho apoio & $4(5,4)$ \\
\hline Lento & $12(16,7)$ & Tenho algum apoio (insuficiente) & $8(10,8)$ \\
\hline Razoável & $15(20,8)$ & Tenho com quem conversar, mas prefiro & $38(51,4)$ \\
\hline Rápido & $29(40,3)$ & não preocupar & \\
\hline Muito rápido & $8(11,1)$ & Não necessito de mais apoio & $24(32,4)$ \\
\hline Esperava esse diagnóstico & & Dinâmica familiar (família nuclear) & \\
\hline Nada & $49(65,3)$ & Mais próxima & $30(40,5)$ \\
\hline Pouco & $10(13,3)$ & Mais afastada & $1(1,4)$ \\
\hline Moderadamente & $9(12)$ & Oscilam períodos de proximidade e & $14(18,9)$ \\
\hline Bastante & $4(5,3)$ & afastamento & \\
\hline Totalmente & $2(2,7)$ & Sem alterações & $29(39,2)$ \\
\hline Evolução da doença & & Dinâmica familiar (família alargada) & \\
\hline Muito lenta & $2(2,7)$ & Mais próxima & $28(37,8)$ \\
\hline Lenta & $8(10,8)$ & Mais afastada & $3(4,1)$ \\
\hline Razoável & $22(29,7)$ & Oscilam períodos de proximidade e & $5(6,8)$ \\
\hline Rápida & $30(40,5)$ & afastamento & \\
\hline Muito rápida & $12(16,3)$ & Sem alterações & $38(5,14)$ \\
\hline Esperava que a doença evoluísse & & Conflitos familiares & \\
\hline desta maneira & & Aumentaram & $7(9,5)$ \\
\hline Nada & $31(42,5)$ & Diminuíram & $4(5,4)$ \\
\hline Pouco & $18(24,6)$ & Oscilaram períodos de mais e menos & $14(18,9)$ \\
\hline Moderadamente & $18(24,6)$ & conflitos & \\
\hline Bastante & $4(5,5)$ & Sem alterações & $49(66,2)$ \\
\hline Totalmente & $2(2,8)$ & & \\
\hline Tempo envolvido nos cuidados & & Relação com o doente & \\
\hline Menos de 3 meses & $9(12)$ & Mais próxima & $49(66,2)$ \\
\hline 3 a 6 meses & $10(13,3)$ & Mais afastada & $3(4,1)$ \\
\hline 7 a 12 meses & $19(25,4)$ & Oscilam períodos de proximidade e & $1(1,4)$ \\
\hline 13 a 24 meses & $10(13,3)$ & afastamento & \\
\hline Mais de 2 anos & $27(36)$ & Sem alterações & $21(28,3)$ \\
\hline Horas por dia dedicadas a & & Tipologia familiar (FRI) & \\
\hline cuidar & & Apoiantes & $12(16)$ \\
\hline Até $2 \mathrm{~h}$ & $7(9,3)$ & Solucionadoras de conflito & $17(22,7)$ \\
\hline $2 \mathrm{a} 4 \mathrm{~h}$ & $7(9,3)$ & Intermédias & $22(29,3)$ \\
\hline 4 a $8 \mathrm{~h}$ & $16(21,4)$ & Mal-humoradas & $18(24)$ \\
\hline 8 a $16 h$ & $14(18,7)$ & Hostis & $6(8)$ \\
\hline \multirow[t]{2}{*}{ Mais de $16 \mathrm{~h}$} & $31(41,3)$ & & \\
\hline & Média (DP) & & Média (DP) \\
\hline Sobrecarga (Zarit) & $33,32(12,53)$ & Sintomatologia Psicopatológica (BSI) & \\
\hline Suporte Social (EPS) & & Ansiedade & $1,33(0,85)$ \\
\hline Apoio Íntimo & $50,93(6,87)$ & Depressão & $1,42(0,80)$ \\
\hline Apoio Casual & $24,48(3,87)$ & Somatização & $0,79(0,70)$ \\
\hline Funcionamento Familiar (FRI) & $8,37(2,52)$ & & \\
\hline
\end{tabular}


renças foram estatisticamente significativas $(\mathrm{U}=$ $318,5 ; \mathrm{W}=1858,5 ; \mathrm{p}=0,012)$. Verificou-se também uma associação negativa entre a sobrecarga e a percepção de apoio prático $(\mathrm{r}=-0,288, \mathrm{p}<$ $0,05)$, de apoio emocional $(r=-0,237, p<0,05)$ e a subescala de apoio íntimo do EPS ( $\mathrm{r}=-0,251$, $\mathrm{p}<0,05)$. A sobrecarga do cuidador apareceu negativamente associada ao estado de saúde do familiar $(\mathrm{r}=-0,281, \mathrm{p}<0,05)$. Por outro lado, estava positivamente associada ao número de horas dedicadas ao cuidado ao doente $(r=0,243$, $\mathrm{p}<0,05)$, à ansiedade $(\mathrm{r}=0,356, \mathrm{p}<0,01)$ e à depressão $(\mathrm{r}=0,315, \mathrm{p}<0,01)$.

A ansiedade $(r=-0,286, p<0,05)$, a somatização $(r=-0,269, \mathrm{p}<0,05)$ e a depressão $(\mathrm{r}=$ $-0,324, \mathrm{p}<0,01)$ mostraram-se negativamente associadas ao apoio emocional recebido, aparecendo também a depressão negativamente associada ao apoio prático $(\mathrm{r}=-0,234, \mathrm{p}<0,05)$. Quando comparados, os cuidadores que já cuidaram de outro doente apresentaram níveis significativamente mais elevados de depressão do que os cuidadores que não cuidaram de outro familiar $(U=442,5 ; W=1523,5 ; p=0,024)$. Foi encontrada uma associação negativa entre a somatização e o tempo que o cuidador está envolvido nos cuidados ao doente $(r=-0,355, p<0,01)$, ou seja, a somatização é maior nos cuidadores que estão a cuidar do doente há menos tempo. No que concerne às variáveis demográficas, verificámos que as mulheres apresentavam níveis significativamente mais elevados de ansiedade $(\mathrm{U}=195 ; \mathrm{W}=300 ; \mathrm{p}=0,001)$, depressão $(\mathrm{U}=$ $184,5 ; \mathrm{W}=289,5 ; \mathrm{p}=0,001)$ e somatização (U $=147 ; \mathrm{W}=252 ; \mathrm{p}<0,01)$. No entanto, através da regressão múltipla, verificamos que as variáveis independentes (percepção de evolução da doença, envolvimento no cuidar, suporte social e dinâmica familiar) têm pouco poder explicativo da sintomatologia depressiva $(11,95 \%)$ e ansiosa $(12,3 \%)$. Apenas a sobrecarga parece contribuir de forma estatisticamente significativa para justificar a variância total da ansiedade $(\mathrm{p}=0,015)$.

Não foi encontrado qualquer tipo de associação significativa entre as diferentes tipologias familiares e a sintomatologia psicopatológica ou a sobrecarga.

\section{Dados obtidos na Fase Inicial do Luto (T1)}

O segundo momento de avaliação decorreu nos primeiros 2 meses de luto dos familiares (tempo médio de luto 55,45 dias, DP = 15,16).

Evolução da sintomatologia psicopatológica Nesta fase de luto, a maioria dos cuidadores apre- sentaram níveis de ansiedade (62\%), depressão (78,6\%) e somatização $(51,7 \%)$ acima da média estabelecida por Canavarro ${ }^{20}$ para a população portuguesa. No Questionário de Experiências Dissociativas Peritraumáticas, a pontuação média foi de 23,69 (DP = 12,16). De acordo com Marmar et al. ${ }^{24}$ uma pontuação total acima de 15 revela experiência de dissociação peritraumática significativa. Na nossa população, 65,5\% dos cuidadores apresentaram valores de dissociação peritraumática clinicamente significativos.

Para a comparação das variáveis avaliadas no T0 e no T1, apenas foram tidos em conta os dados relativos aos 29 sujeitos que responderam aos dois momentos. Verificamos que os valores médios da depressão, ansiedade e somatização reduziram ligeiramente no segundo momento de avaliação; no entanto, as diferenças não foram consideradas estatisticamente significativas. No que diz respeito à percepção da sobrecarga, reconheceram-se diferenças significativas entre o T0 $(\mathrm{M}=1,64 ; \mathrm{SEM}=0,268)$ e o $\mathrm{T} 1(\mathrm{M}=2,21$; SEM $=0,264,(\mathrm{t}(28)=-2,46 ; \mathrm{p}=0,021,2 \mathrm{p}=0,183$, potência $(\varpi)=0,659)$, ou seja, na fase de luto, os cuidadores fazem uma avaliação retrospectiva de maior sobrecarga do que aquela referida durante a fase de prestação de cuidados. Comparando a percepção de suporte social, não se verificaram diferenças significativas entre os dois momentos.

Descrição dos fatores situacionais - A maioria (55,2\%) dos doentes faleceu no Hospital de Santa Maria, 24,1\% faleceu em casa, e 44,8\% dos cuidadores familiares estiveram presentes no momento do óbito. Quase todos os cuidadores (89,7\%) relataram ter bastante conhecimento sobre a gravidade da doença e 89,6\% referiram que os profissionais de saúde falaram sobre a proximidade da morte do seu familiar em algum momento. Apesar de avisados sobre a iminência da morte, 31\% dos cuidadores disseram estar pouco preparados para a perda do seu ente querido. Questionados sobre a qualidade dos cuidados prestados ao doente pelos profissionais de saúde, 37,9\% dos cuidadores avaliaram como razoável e a mesma percentagem avaliou como muito bom (Tabela 4).

Grande parte dos cuidadores (87,5\%) referiu ter apoio no luto, principalmente da família $(62,5 \%), 55,2 \%$ dos cuidadores referiu não necessitar de mais apoio prático e $62,1 \%$ referiu ter com quem conversar, mas que preferia não os preocupar com seus problemas. Relativamente à dinâmica familiar, não houve alterações para a grande maioria dos participantes (Tabela 4).

Fatores relacionados com a sintomatologia psicopatológica - A experiência de dissociação 
Tabela 4. Variáveis dependentes e independentes avaliadas no T1.

\begin{tabular}{|c|c|c|c|}
\hline Variável & $\mathrm{N}=29$ & Variável & $\mathbf{N}=29$ \\
\hline Conhecimento sobre a gravidade da doença & n (\%) & Apoio prático & n (\%) \\
\hline Moderadamente & $3(10,3)$ & Necessitava de mais apoio & $3(10,3)$ \\
\hline Bastante & $14(48,3)$ & Tenho algum apoio & $10(34,5)$ \\
\hline \multirow[t]{2}{*}{ Extremamente } & $12(41,4)$ & (insuficiente) & \\
\hline & & Não necessito de mais apoio & $16(55,2)$ \\
\hline Avisado sobre a proximidade da morte & & Apoio emocional & \\
\hline Nada (Não) & $3(10,4)$ & Necessitava de mais apoio & 0 \\
\hline Poucas vezes & $6(20,7)$ & Tenho algum apoio & $3(10,3)$ \\
\hline Algumas vezes & $7(24,1)$ & (insuficiente) & \\
\hline Muitas vezes & $7(24,1)$ & Tenho com quem conversar, mas & $18(62,1)$ \\
\hline \multirow[t]{2}{*}{ Sempre } & $6(20,7)$ & prefiro não preocupar & \\
\hline & & Não necessito de mais apoio & $8(27,6)$ \\
\hline \multicolumn{2}{|l|}{ Estava preparado emocionalmente para a perda } & Dinâmica familiar (família nuclear) & \\
\hline Nada & $3(10,3)$ & Mais próxima & $6(20,7)$ \\
\hline Pouco & $9(31)$ & Mais afastada & $3(10,3)$ \\
\hline Moderadamente & $4(13,8)$ & Oscilam períodos de & $6(20,7)$ \\
\hline Bastante & $9(31)$ & proximidade e afastamento & \\
\hline Extremamente & $4(13,8)$ & Sem alterações & $14(48,3)$ \\
\hline \multicolumn{2}{|l|}{ Como avalia os cuidados prestados ao doente } & \multicolumn{2}{|l|}{ Dinâmica familiar (família alargada) } \\
\hline Muito mau & $1(3,5)$ & Mais próxima & $6(20,7)$ \\
\hline Mau & $2(6,9)$ & Mais afastada & $2(6,9)$ \\
\hline Razoável & $11(37,9)$ & Oscilam períodos de & $7(24,1)$ \\
\hline Bom & $4(13,8)$ & proximidade e afastamento & \\
\hline Muito bom & $11(37,9)$ & Sem alterações & $14(48,3)$ \\
\hline \multicolumn{2}{|c|}{$\begin{array}{l}\text { Como avalia o seu nível de sobrecarga durante o período } \\
\text { que cuidou do doente }\end{array}$} & Conflitos familiares & \\
\hline Nada & $13(46,4)$ & Aumentaram & $3(10,3)$ \\
\hline Um pouco & $5(17,9)$ & Diminuíram & $1(3,4)$ \\
\hline Moderado & $5(17,9)$ & Oscilaram períodos de mais e & $4(13,8)$ \\
\hline Bastante & $2(7,1)$ & menos conflitos & \\
\hline \multirow[t]{2}{*}{ Extremamente } & $3(10,7)$ & Sem alterações & $21(72,4)$ \\
\hline & Média (DP) & & Média (DP) \\
\hline Tempo de luto (dias) & $55,45(15,16)$ & Sintomatologia Psicopatológica (BSI) & \\
\hline \multirow{3}{*}{$\begin{array}{l}\text { Experiência Dissociativa Peritraumática } \\
\text { (QEDP) }\end{array}$} & $23,69(12,16)$ & Ansiedade & $1,36(0,95)$ \\
\hline & & Depressão & $1,44(0,85)$ \\
\hline & & Somatização & $0,81(0,82)$ \\
\hline
\end{tabular}

peritraumática estava positivamente associada à ansiedade $(\mathrm{r}=0,668, \mathrm{p}<0,01)$, à depressão ( $\mathrm{r}$ $=0,429, \mathrm{p}<0,05)$ e à somatização $(\mathrm{r}=0,548, \mathrm{p}$ $<0,01)$. A percepção de apoio prático apareceu negativamente associada à depressão $(\mathrm{r}=-0,609$, $\mathrm{p}<0,01)$, ansiedade $(\mathrm{r}=-0,451, \mathrm{p}<0,05)$, e somatização $(r=-0,572, p<0,01)$. O suporte social prático mostrou-se negativamente relacionado com a depressão $(\mathrm{r}=-0,714, \mathrm{p}<0,01)$ e somatização no luto $(r=-0,503, p<0,05)$. O suporte emocional estava associado a valores mais baixos de depressão $(r=-0,418, p<0,05)$.

Ponderando a influência das circunstâncias anteriores à morte, verificou-se que o tempo de diagnóstico estava inversamente correlaciona- do com os níveis de depressão $(\mathrm{r}=-0,416, \mathrm{p}<$ $0,05)$ e de somatização $(\mathrm{r}=0,409, \mathrm{p}<0,05)$. Os cuidadores que reponderam que não estavam à espera que a doença evoluísse daquela maneira são aqueles que maior ansiedade $(r=-0,452, p$ $<0,05)$ e sintomas peritraumáticos $(\mathrm{r}=-0,43$, $\mathrm{p}$ $<0,05)$ apresentam na fase de luto. No entanto, outras variáveis relacionadas com a percepção subjectiva de preparação para a morte não interferem na sintomatologia psicopatológica dos cuidadores no período de luto.

Já a sobrecarga do cuidador está associada com a percepção de estar emocionalmente preparado para a morte $(\mathrm{r}=0,408, \mathrm{p}<0,05)$ e não parece influenciar os níveis de sintomatologia 
psicopatológica no período posterior à morte. A avaliação retrospectiva de sobrecarga está negativamente correlacionada com o nível de somatização no luto $(r=-0,424, p<0,05)$. No que se refere ao suporte social, as pessoas que relataram mais apoio prático na fase anterior à perda, apresentam valores mais elevados de sintomas peritraumáticos $(\mathrm{r}=0,575, \mathrm{p}<0,01)$ e de ansiedade $(\mathrm{r}=0,534, \mathrm{p}<0,01)$ no luto agudo.

\section{Discussão}

O presente estudo teve, como primeiro objetivo, caracterizar os cuidadores familiares portugueses dos doentes acompanhados em cuidados paliativos em relação aos níveis de sobrecarga e sintomas psicopatológicos, reconhecendo a influência das circunstâncias que afetam o papel do cuidador, designadamente a percepção da evolução da doença, envolvimento no cuidar, percepção de suporte social e dinâmica familiar.

À semelhança da grande parte dos estudos que envolvem cuidadores ${ }^{26-28}$, a amostra é maioritariamente constituída por mulheres, casadas e filhas do doente. Uma parte significativa dos cuidadores $(78,7 \%)$ apresentava uma sobrecarga moderada a severa. Contribuem para a sensação de sobrecarga do cuidador o tempo dispendido nos cuidados ao doente, sendo que $41,3 \%$ dedicavam mais de 16 horas do seu dia a cuidar do seu familiar. Em consonância com este resultado, Brazil et al. ${ }^{29}$ referem que os familiares que passavam a maior parte do dia a cuidar do doente apresentavam maior risco de elevada sobrecarga. Outro aspecto que aparece associado à sobrecarga é a percepção de pior estado de saúde do doente. Grunfeld et al. ${ }^{30}$ corroboram que a sobrecarga percebida nos cuidadores aumentava com o declínio do estado funcional dos pacientes.

Aqueles com maiores níveis de sobrecarga são também aqueles que têm menos suporte social, nomeadamente nas formas de apoio prático, emocional e íntimo. Estes resultados são consistentes com outros estudos que detectaram elevados níveis de sobrecarga na população de cuidadores de doentes oncológicos em estádio avançado ou em cuidados paliativos ${ }^{31,32}$, e como no estudo de Salmon et al..$^{33}$ que encontrou uma associação entre menores níveis de sobrecarga e a percepção de apoio de outras pessoas.

De salientar, porém, que muitos cuidadores reconhecem que, desde que se manifestou a doença, a família está mais próxima e que existe disponibilidade de suporte emocional, mas prefe- rem não solicitar esse apoio para evitar sobrecarregar as outras pessoas. Este dado coincide com a observação de que, durante esta fase, a expressão emocional tende a ser reprimida pelo facto de os cuidadores terem a percepção de que toda a família está sob estresse ${ }^{34,35}$.

Os cuidadores que têm maiores níveis de sobrecarga são também aqueles que têm mais depressão e ansiedade ${ }^{30,36}$. Este tipo de sintomatologia é muito encontrado na população de cuidadores de doentes em fim de vida ${ }^{32,37}$. O nosso estudo corrobora a elevada prevalência de sintomas de depressão, ansiedade e somatização na população portuguesa de cuidadores e a sua associação com a sobrecarga e a falta de apoio. Especificamente, a falta de apoio emocional está associada às três medidas de sintomatologia psicopatológica, enquanto a escassez de apoio prático e o facto de terem cuidado anteriormente de outro doente aparecem relacionadas, em particular, com a depressão. Do nosso conhecimento, este é o primeiro estudo que discrimina de que forma as falhas de suporte emocional e prático interferem nas diversas formas de sintomatologia psicopatológica dos cuidadores em cuidados paliativos.

Verificou-se, através da análise de regressão que, do conjunto dos fatores situacionais, só a sobrecarga contribui significativamente para a ansiedade, o que pode ser explicado pela elevada ambivalência dos cuidadores face às próprias necessidades e na gestão das exigências do cuidar ${ }^{38,39}$. Por outro lado, a somatização parece prevalecer numa fase inicial da prestação de cuidados, remetendo para a elevada reatividade fisiológica associada ao impacto das tarefas do cuidar.

Em resposta ao segundo objetivo, propusemo-nos avaliar a evolução dos sintomas psicopatológicos, identificando os fatores circunstanciais que interferem na fase aguda do luto. Os resultados sustentam a hipótese de continuidade das manifestações de ansiedade, depressão e somatização, como está descrito na literatura ${ }^{15}$. Nesta fase, foram avaliados também os sintomas peritraumáticos, que atingem valores clinicamente significativos em 65,5\% dos participantes, estando associados com todas as formas de sintomatologia psicopatológica. Corrobora-se assim a existência de uma importante componente traumática nas manifestações de luto dos cuidadores ${ }^{16}$.

Das circunstâncias da doença, o tempo de diagnóstico breve é o único fator que surge associado à depressão e somatização no luto. Os sintomas de trauma e de ansiedade estão sobretudo relacionados com o facto de os cuidadores não 
estarem à espera que a doença evoluísse daquela forma, o que aconteceu em 42,5\% dos casos. Tem sido documentado que, na perda por doença avançada, a falta de preparação para a morte, resultante de uma insuficiente comunicação, está associada a mais manifestações no luto ${ }^{40}$. De salientar, no entanto, que esta avaliação, realizada numa fase inicial do acompanhamento em cuidados paliativos, não se traduziu, posteriormente, na percepção de falta de informação, uma vez que $89,6 \%$ dos familiares referiram que os profissionais de saúde falaram sobre a proximidade da morte do seu familiar em algum momento. Não obstante, a avaliação que o familiar faz da preparação subjectiva para a morte subsequente à morte não exerce influência na sintomatologia psicopatológica, o que permite concluir que esta avaliação retrospectiva pode não aferir o impacto real desta dimensão no luto.

Mas o dado mais surpreendente deste estudo talvez seja o facto de, embora na fase de luto, os cuidadores tendam a desvalorizar a exaustão que experimentaram durante a fase de prestação de cuidados, o valor da sobrecarga é superior àquele antes referido. É expectável que este valor acrescido se deva ao efeito cumulativo das circunstâncias do cuidar e da perda. No entanto, o nível de sobrecarga percebido na fase de prestação de cuidados não parece ter um efeito significativo na sintomatologia psicopatológica posterior à perda. Já a avaliação retrospectiva de sobrecarga está negativamente correlacionada com o nível de somatização no luto. Possivelmente estes resultados estão relacionados com a influência da sobrecarga na percepção de estar emocionalmente preparado para a morte, ou seja, quando o cuidador se sente sobrecarregado com as tarefas de assistência ao doente, acaba por antecipar a morte como um alívio para o sofrimento de ambos, e a recordação desta experiência atenua a experiência de distress ao nível psicofisiológico.Significa isto que a sobrecarga do cuidador poderá não ter um impacto direto sobre o luto: contribui para a sintomatologia apenas na fase de prestação de cuidados e esta tem tendência a manter-se na fase posterior à perda.

No que respeita à percepção do suporte social no luto, verificamos que a maior parte das pes- soas refere a disponibilidade de apoio emocional e prático e este facto parece ter uma influência positiva na redução dos sintomas depressivos no luto agudo, como tem sido referido na literatura ${ }^{41}$.

\section{Conclusões}

Dada a inexistência de dados relativos à população portuguesa, este estudo torna-se importante por dar a conhecer os elevados níveis de morbilidade psicológica dos cuidadores familiares acompanhados em cuidados paliativos e as circunstâncias que os afetam. Baseados nestes resultados, é possível reconhecer que as pessoas mais envolvidas nos cuidados ao doente apresentam mais sobrecarga e estão também mais propensas à sintomatologia psicopatológica. Esta tem tendência a manter-se na fase aguda de luto, pelo que ficou demonstrada a continuidade das manifestações de distress no período de luto agudo. Este efeito deve ser alvo de futura investigação. Outro dado importante que resulta desta investigação é a confirmação de que esta população experimenta sintomas peritraumáticos que parecem contribuir significativamente para o estado geral de distress no luto agudo. Por fim, há que considerar que as formas de apoio prático e emocional têm efeito protetor específico nos diferentes quadros sintomatológicos e estão estreitamente relacionadas com o funcionamento das famílias. Estes resultados têm evidentes implicações clínicas na identificação precoce das situações de maior vulnerabilidade e construção de programas de intervenção dirigidos às necessidades específicas desta população.

A mais importante limitação do estudo prende-se com a amostra reduzida, sobretudo no T1, o que coloca reservas à generalização dos resultados. O número insuficiente de participantes no segundo momento inviabilizou a realização de análises de regressão, o que condiciona o tratamento dos resultados. É possível também que a seleção da amostra esteja enviesada, e eventualmente apresente níveis de instrução superiores relativamente aos que não participaram, tendo em conta que são maioritariamente filhos, com idades inferiores relativamente à população de cônjuges. 


\section{Colaboradores}

M Delalibera, A Coelho, J Presa foram responsáveis pela concepção e elaboração da pesquisa, análise dos dados e redação do texto. A Barbosa e I Leal colaboraram na revisão crítica do texto.

\section{Agradecimentos}

À Coordenação de Aperfeiçoamento de Pessoa de Nível Superior (CAPES) uma vez que, este trabalho foi desenvolvido no âmbito do doutorado no exterior da autora principal, como bolsista CAPES.

\section{Referências}

1. Schulz R, Beach SR, Lind B, Martire LM, Zdaniuk B, Hirsch C, Jackson S, Burton L. Involvement in caregiving and adjustment to death of a spouse: findings from the caregiver health effects study. Jama 2001; 285(24):3123-3129.

2. Kim Y, Baker F, Spillers RL, Wellisch DK. Psychological adjustment of cancer caregivers with multiple roles. Psychooncology 2006; 15(9):795-804.

3. Kissane DW, Bloch S, Burns WI, McKenzie D, Posterino M. Psychological morbidity in the families of patients with cancer. Psychooncology 1994; 3(1):47-56.

4. Kim Y, Schulz R. Family caregivers' strains: Comparative analysis of cancer caregiving with dementia, diabetes, and frail elderly caregiving. J Aging Health 2008; 20(5):483-503.

5. Given B, Wyatt G, Given C, Gift A, Sherwood P, DeVoss $\mathrm{D}$, Rahbar M. Burden and depression among caregivers of patients with cancer at the end-of-life. Oncol Nurs Forum 2004; 31(6):1105-1117.

6. Grov EK, Dahl AA, Moum T, Fosså SD. Anxiety, depression, and quality of life in caregivers of patients with cancer in late palliative phase. Ann Oncol 2005; 16(7):1185-1191.

7. Burton AM, Haley WE, Small BJ, Finley MR, Dillinger-Vasille M, Schonwetter R. Predictors of well-being in bereaved former hospice caregivers: The role of caregiving stressors, appraisals, and social resources. Palliat Support Care 2008; 6(02):149-158.

8. Abernethy A, Burns C, Wheeler J, Currow D. Defining distinct caregiver subpopulations by intensity of endof-life care provided. Palliat Med 2008; 23(1):66-79.

9. Barry LC, Kasl SV, Prigerson HG. Psychiatric disorders among bereaved persons: the role of perceived circumstances of death and preparedness for death. Am J Geriatr Psychiatry 2002; 10(4):447-457.

10. Kang J, Shin DW, Choi JE, Sanjo M, Yoon SJ, Kim HK, Oh MS, Kwen HS, Choi HY, Yoon WH. Factors associated with positive consequences of serving as a family caregiver for a terminal cancer patient. Psychooncology 2013; 22(3):564-571.

11. Nijboer C, Tempelaar R, Triemstra M, van den Bos GA, Sanderman R. The role of social and psychologic resources in caregiving of cancer patients. Cancer 2001; 91(5):1029-1039.

12. Proot IM, Abu-Saad HH, Crebolder HFJM, Goldsteen M, Luker KA, Widdershoven GAM. Vulnerability of family caregivers in terminal palliative care at home; balancing between burden and capacity. Scand J Caring Sci 2003; 17(2):113-121.

13. Kissane DW, Bloch S, Onghena P, McKenzie DP, Snyder RD, Dowe DL. The Melbourne Family Grief Study, II: Psychosocial morbidity and grief in bereaved families. Am J Psychiatry 1996; 153(5):659-666.

14. Thomas K, Hudson P, Trauer T, Remedios C, Clarke D. Risk factors for developing prolonged grief during bereavement in family carers of cancer patients in palliative care: a longitudinal study. J Pain Symptom Manage 2014; 47(3):531-541.

15. Kissane DW, Bloch S, Burns WI, Patrick JD, Wallace CS, McKenzie DP. Perceptions of family functioning and cancer. Psychooncology 1994; 3(4):259-269. 
16. Chentsova-Dutton Y, Shucter S, Hutchin S, Strause L, Burns K, Dunn L, Miller M, Zisook S. Depression and grief reactions in hospice caregivers: From pre-death to 1 year afterwards. J Affect Disord 2002; 69(1):53-60.

17. Sanderson C, Lobb EA, Mowll J, Butow PN, McGowan N, Price M A. Signs of post-traumatic stress disorder in caregivers following an expected death: A qualitative study. Palliat Med 2013; 27(7):625-631.

18. Ferreira F, Pinto A, Laranjeira A, Pinto AC, Lopes A, Viana A, Rosa B, Esteves C, Pereira I, Nunes I, Miranda J, Fernandes P, Miguel S, Leal V, Fonseca C. Validação da escala de Zarit: sobrecarga do cuidador em cuidados paliativos domiciliários, para população portuguesa. Cad Saude 2010; 3(2):13-19.

19. Derogatis LR, Melisaratos N. The brief symptom inventory: an introductory report. Psychol Med 1983; 13(3):595-605.

20. Canavarro MC. Inventário de sintomas psicopatológicos: BSI. In: Simões MR, Gonçalves M, Almeida LS. editores. Testes e provas psicológicas em Portugal. Braga: SHO/APPORT; 1999. p. 87-109.

21. Kissane DW, Bloch S, McKenzie M, McDowall AC, Nitzan R. Family grief therapy: a preliminary account of a new model to promote healthy family functioning during palliative care and bereavement. Psychooncology 1998; 7(1):14-25.

22. Delalibera M, Coelho A, Barbosa A, Leal I. Contributos para a validação portuguesa do Family Relationship Index (FRI) reduzido. (in press).

23. Moreira JM, Canaipa RITA. A Escala de Provisões Sociais: Desenvolvimento e validação da versão portuguesa da "Social Provisions Scale". Rev Iberoam Diagn Ev 2007; 24(2):23-58.

24. Marmar C, Weiss D, Metzler T. The peritraumatic dissociative experience questionnaire. In: Wilson J, Keane T, editors. Assessing Psychological Trauma and PTSD. New York: The Guilford Press; 1997. p. 412-428.

25. Maia A, Moreira S, Fernandes E. Adaptação para a língua portuguesa do Questionário de Experiências Dissociativas Peritraumáticas (QEDP) numa amostra de bombeiros. Rev Psiquiatr Clin 2009; 36(1):1-9.

26. Hudson PL, Thomas K, Trauer T, Remedios C, Clarke D. Psychological and social profile of family caregivers on commencement of palliative care. J Pain Symptom Manage 2011; 41(3):522-534.

27. Kapari M, Addington-Hall J, Hotopf, M. Risk factors for common mental disorder in caregiving and bereavement. J Pain Symptom Manage 2010; 40(6):844856.

28. Presa J. Género e processo de luto. In: Barbosa A, Coelho A, editores. Mediadores do Luto. Lisboa: Núcleo Académico de Estudos e Intervenção sobre Luto, Centro de Bioética da Faculdade de Medicina da Universidade de Lisboa; 2014. p. 21-41.

29. Brazil K, Bédard M, Willison K, Hode M. Caregiving and its impact on families of the terminally ill. Aging Ment Health 2003; 7(5):376-382.

30. Grunfeld E, Coyle D, Whelan T, Clinch J, Reyno L, Earle CC, Willan A, Viola R, Coristine M, Janz T, Glossop R. Family caregiver burden: results of a longitudinal study of breast cancer patients and their principal caregivers. Can Med Assoc J 2004; 170(12):1795-1801.
31. Braun M, Mikulincer M, Rydall A, Walsh A, Rodin G. Hidden morbidity in cancer: spouse caregivers. J Clin Oncol 2007; 25(30):4829-4834

32. Costa-Requena G, Cristófol R, Cañete J. Caregivers' morbidity in palliative care unit: predicting by gender, age, burden and self-esteem. Support Care Cancer 2012; 20(7):1465-1470.

33. Salmon JR, Kwak J, Acquaviva KD, Brandt K, Egan KA. Transformative aspects of caregiving at life's end. J Pain Symptom Manage 2005; 29(2):121-129.

34. Saldinger A, Cain AC. Deromanticizing anticipated death: Denial, disbelief and disconnection in bereaved spouses. J Clin Oncol 2005; 22(3):69-92.

35. Beng TS, Guan NC, Seang LK, Pathmawathi S, Ming MF, Jane LE, Chin LE, Loong LC. The Experiences of Suffering of Palliative Care Informal Caregivers in Malaysia A Thematic Analysis. Am J Hosp Palliat Medicine Care 2013; 30(5):473-489.

36. Castillo OIA, Morales-Vigil T, Vázquez-Pineda F, Sánchez-Román S, Ramos-del Río B, Guevara-López U. Sobrecarga, ansiedad y depresión en cuidadores primarios de pacientes con dolor crónico y terminales. Rev Med Inst Mex Seguro Soc 2008; 46(5):485-495.

37. Wasner M, Paal P, Borasio GD. Psychosocial care for the caregivers of primary malignant brain tumor patients. J Soc Work End Life Palliat Care 2013; 9(1):74-95.

38. Harding R, Higginson I. Working with ambivalence: informal caregivers of patients at the end of life. Support Care Cancer 2001; 9(8):642-645.

39. Coelho A. A Experiência Antecipada da Perda e o Processo de Luto. In: Barbosa A, editor. Contextos do Luto. Lisboa: Núcleo Académico de Estudos e Intervenção no Luto, Centro de Bioética, Faculdade de Medicina da Universidade de Lisboa; 2014. p. 31-64.

40. Wright AA, Zhang B, Ray A, Mack JW, Trice E. Balboni T, Mitchell SL, Jackson VA, Block SD, Prigerson HG. Associations between end-of-life discussions, patient mental health, medical care near death, and caregiver bereavement adjustment. Jama 2008; 300(14):16651673.

41. Stroebe W, Zech E, Stroebe MS, Abakoumkin G. Does Social Support Help in Bereavement? J Soc Clin Psychol 2005; 24(7):1030-1050.

Artigo apresentado em 18/04/2016

Aprovado em 02/08/2016

Versão final apresentada em 04/08/2016 